\title{
ESTRATEGIAS COGNITIVO-EMOCIONALES Y DENSIDAD ARGUMENTATIVA ${ }^{1}$ \\ EMOTIONAL COGNITIVE STRATEGIES AND ARGUMENTATIVE DENSITY
}

\author{
CRISTIÁN NOEMI \\ Universidad de La Serena \\ cnoemi@userena.cl \\ ROSALÍA IGLESIAS \\ Universidad de La Serena \\ neftis32@hotmail.com
}

\section{RESUMEN}

El trabajo procura establecer si los modos con los que un sujeto se enfrenta cognitiva y emocionalmente con la realidad están relacionados con su conducta lingüística expresada en términos de densidad argumentativa. A fin de establecer cuáles son las principales estrategias cognitivo-emocionales empleadas por los sujetos de la muestra, se empleó el test Moldes (Hernández, 2010). Para evaluar la densidad discursiva argumentativa se utilizó el Dilema III del Cuestionario D.I.T. (Rest, 2013). El cruce de información entre ambos instrumentos permitió establecer que los sujetos de alto rendimiento en el test Moldes mostraron mayor grado de densidad discursiva y viceversa. Los resultados, igualmente, permitieron especificar los tipos de estrategias cognitivo-emocionales que se asocian con mayor o menor densidad discursiva argumentativa.

Palabras clave: Estrategias cognitivas, estrategias emocionales, densidad argumentativa, densidad discursiva.

\section{ABSTRACT}

The work tries to establish whether the ways in which a subject is confronted cognitively and emotionally with reality are related to their linguistic behavior expressed in terms of argumentative density. In order to establish which are the main cognitive emotional strate-

${ }^{1}$ Este trabajo se enmarca en el proyecto de investigación FONDECYT No 1170492 y DIDULS PR16211. 
gies used by the subjects of the sample, the Moldes test was used (Hernández, 2010). To assess the argumentative discursive density, the Dilemma III of the Questionnaire D.I.T. (Rest, 2013) was used. The crossing of information between both instruments allowed to establish that the subjects of high performance in the Molds test showed a greater degree of discursive density and vice versa. The results also allowed us to specify the types of cognitive emotional strategies that are associated with greater or lesser argumentative discursive density.

Keywords: Cognitive strategies, emotional strategies, argumentative density, discursive density.

Recibido: 07/08/2018. Aceptado: 28/05/2019.

\section{INTRODUCCIÓN}

i bien la teoría de la argumentación ha tenido en las últimas décadas un avance $\checkmark$ conceptual considerable, existe todavía un gran vacío en lo que respecta al conocimiento de la ontogénesis de esta capacidad (Santibánez, 2016), y particularmente en lo que guarda relación con variables psicosociales que permitan explicar su naturaleza en cuanto facultad.

A fin de contribuir en este sentido, el trabajo devela aspectos de la relación entre las estrategias cognitivo-emocionales empleadas por estudiantes universitarios chilenos al realizar una tarea de escritura, y el nivel o grado de densidad textual argumentativo logrado en sus productos discursivos.

En términos generales, esta relación ha sido sólo superficialmente explorada, considerando, a su vez, un conjunto muy limitado de variables intervinientes, y poniendo mayor énfasis sobre la estructura de superficie textual, particularmente respecto de la organización sintáctica (Véliz, 1999; Van Eemeren, Grootendorst y Snoeck, 2006).

En efecto, si bien existen investigaciones que abordan la problemática de la argumentación en estudiantes universitarios (Marinkovich, 2007; Padilla, Douglas y López, 2010), los estudios que se focalizan sobre el vínculo competencia/ actuación en este sentido son casi inexistentes.

Los trabajos de Hample (2005) y Hample, Warner y Young (2009), orientados a establecer las preconcepciones (esquemas) asociadas a la actividad argumentativa en estudiantes universitarios, constituyen una excepción al respecto; lo propio puede decirse del aporte de Jélvez (2008), focalizado sobre el empleo de esquemas argumentativos en estudiantes secundarios, o el de Marinkovich (2007), orientado a establecer la naturaleza de este tipo de competencia, ambos en el contexto de habla hispánica.

En este tenor, el trabajo ha pretendido determinar el tipo de estrategias cog- 
nitivo-emocionales (moldes mentales) que se asocian a la producción discursiva argumentativa de estudiantes universitarios y las características formales de los constructos lingüísticos generados. El objetivo principal ha pretendido describir los tipos de estrategias cognitivo-emocionales empleadas por este tipo de hablantes al enfrentar una tarea escritural, y develar su relación con el grado de densidad argumentativa alcanzado en sus productos discursivos.

La pretensión por aproximarse a la competencia involucrada en este tipo de productos discursivos se inscribe en el ámbito de los estudios cognitivos, toda vez que se intenta parcialmente referir en definitiva tipos de representaciones mentales asociadas con productos discursivos determinados (Tomasello, 1999).

Si consideramos que los moldes mentales son modos habituales con los que una persona se enfrenta cognoscitiva y afectivamente a la realidad, y con los que interpreta y valora su relación con ella, su valor funcional establece la pertinencia de evaluar determinadas conductas lingüísticas asociadas (Capote, Fernández y Hernández, 2007).

Para estos propósitos, en la sección Marco Teórico se proponen, refieren y discuten las nociones de molde mental y densidad argumentativa. En la sección Metodología, se da cuenta del tipo de diseño del trabajo y se caracteriza la muestra del corpus. En el apartado Análisis se ofrecen los resultados obtenidos luego de la aplicación del test Moldes y del Dilema III del Cuestionario D.I.T. En la sección Hallazgos se presentan y comentan las principales tendencias que se observan luego del análisis del corpus, y finalmente en el apartado Conclusión se refieren los principales alcances y limitaciones del trabajo.

\section{MARCO TEÓRICO}

\subsection{Moldes mentales}

Se concibe como moldes mentales (o estrategias cognitivo-emocionales) aquellos patrones de pensamiento habituales y peculiares que orientan o guían la interpretación de la realidad de las personas en situaciones de egoimplicación, es decir, donde se ven enfrentadas a un contexto que compromete sus intereses y emociones (Hernández, 2010).

Preferentemente, las estrategias cognitivo-emocionales han sido abordadas para intentar explicar conductas tales como la inadaptación social, el bienestar subjetivo, la ansiedad, la esquizofrenia, los estilos educadores de los padres, o el rendimiento académico, por nombrar algunas (Capote, García, y Hernández, 2002; Hernández, 2005; Hernández y Rodríguez, 2006; Hernández, 2006; Hernández, 2007; Capote et al., 2007).

Específicamente, la noción de molde mental se asocia al paradigma emocio- 
nal-constructivista (Hernández, 1991; Hernández, 2002), postura que propone incluir las emociones para dar cuenta del modo en que los sujetos estructuran cognitivamente el contexto.

En esta línea de pensamiento, Beck (1974) postuló originalmente la existencia de un 'esquema', esto es, un filtro o plantilla mental con la cual las personas abordarían diferentes situaciones. El esquema, en este contexto, se concibió como una suerte de significado idiosincrásico a partir del cual los sujetos otorgarían sentido a las diferentes situaciones vitales con las que se enfrentan (Beck, 1974).

Contemporáneamente el concepto de esquema ha sido tratado básicamente desde dos perspectivas; en primer lugar, asociando la noción al concepto clásico de topos, i.e., una aproximación de orden más bien cognitivo; o bien, por otra parte, desde una perspectiva basada en un dominio de naturaleza pragmática.

Hastings (1963), por ejemplo, desde la primera óptica describe nueve modos de razonamiento que agrupa en tres clases: procedimientos verbales y semánticos; conexiones causales; y argumentos soportados ya sea en conclusiones verbales o causales (Hastings, 1963). En un sentido similar, Perelman concibe la noción de topos, ya como premisas de argumentos o bien como depósito de argumentos que agrupa en categorías tales como lugares de la cantidad, de la cualidad, del orden, de lo existente, de la esencia, o de la persona (Perelman y Olbrechts, 1989). En tanto, Kienpointner (1987), en esa misma línea, propone una clasificación de esquemas de argumentos en relación con reglas de generalización, a partir de opiniones socialmente aceptadas (Kienpointner, 1987).

Por otro lado, desde una perspectiva pragmática, Walton, Reed y Macagno (2008), proponen una categorización de esquemas en contextos conversacionales específicos, a partir del establecimiento de posibles falacias, las que en su opinión no se determinan simplemente a partir de la información vehiculada proposicionalmente, sino que también y fundamentalmente, por la situación contextual y dialogal específica, tal cual es el caso, por ejemplo, del argumento de autoridad (Walton, 1997).

Con independencia del modo de aproximación, ambas posturas coinciden en que, cualquiera sea el caso, la noción de esquema supone procesos de inferencias (textuales o pragmáticas) que modelan la estructura de los tipos de argumentos (Walton, 1996; 2005). Desde la primera perspectiva se propone que las inferencias basadas en el lenguaje se generan más bien de manera automática, y que estas son funcionalmente independientes del conocimiento previo del lector (Graesser, Singer y Trabasso, 1994). Desde la segunda, se sugiere que el conocimiento previo del sujeto interactúa con el procesamiento del lenguaje a través de inferencias que dependen del conocimiento del mundo que el sujeto posea (Van Eemeren, 2012).

En lo particular, el modelo de moldes mentales (Hernández, 1991; Hernán$\mathrm{dez}, 2002)$ propone que los individuos poseen un mapa o sistema innato de representaciones (que se expresa en tres encuadres), el que para poder ser activado 
(a través de diez dimensiones) requiere de una actualización (por medio de treinta moldes mentales), dependiendo de necesidades cognitivas puntuales.

De acuerdo con la propuesta de Hernández, los moldes son conformados por los propios sujetos como consecuencia de sus predisposiciones y experiencias, por lo que pueden caracterizarse como constructos derivados de la interacción entre predisposiciones genéticas, por una parte, y el aprendizaje proveniente de los diferentes entornos a los que nos vemos expuestos en la experiencia cotidiana, por otra (Hernández, 1991, 2010).

Para la consecución empírica de los moldes mentales (ver Tabla I), a través de análisis factorial, Hernández (2002) obtuvo treinta moldes, los que se engloban en diez dimensiones y en tres encuadres focales.

Tabla I. Estructura jerárquica del test Moldes* (*ver Anexo 1. Especificación Moldes)

\begin{tabular}{|c|c|c|}
\hline Encuadres & Dimensiones & Moldes \\
\hline \multirow{3}{*}{$\begin{array}{l}\text { E1 Espontaneidad } \\
\text { vital }\end{array}$} & \multirow{3}{*}{ D1 Implicación vital } & M1 Implicancia directa \\
\hline & & M2 Hiperanálisis \\
\hline & & M3 Hipercontrol anticipatorio \\
\hline \multirow{19}{*}{ E2 Ajuste } & \multirow{3}{*}{ D2 Negatividad } & M5 Anticipación aversiva \\
\hline & & M6 Evaluación Selectiva negativa \\
\hline & & M7 Hostiligencia \\
\hline & \multirow{4}{*}{ D3 Discordia } & M4 Afrontamiento borroso \\
\hline & & M8 Inflación-decepción \\
\hline & & M9 Focalización en carencias \\
\hline & & M10 Imantación por lo imposible \\
\hline & \multirow{4}{*}{ D4 Evitación } & M11 Anticipación devaluativa \\
\hline & & M12 Disociación emocional \\
\hline & & M13 Reclusión \\
\hline & & M14 Oblicuidad cognitiva \\
\hline & \multirow{2}{*}{ D5 Defensividad } & M18 Justificación de los fallos \\
\hline & & M19 Desplazamiento emocional \\
\hline & \multirow{3}{*}{$\begin{array}{l}\text { D6 Atribución } \\
\text { externa }\end{array}$} & M20 Atribución al temperamento \\
\hline & & M21 Atribución social del éxito \\
\hline & & M22 Atribución mágica \\
\hline & \multirow{3}{*}{ D7 Operatividad } & M15 Anticipación esfuerzo-costo \\
\hline & & M16 Precisión y supervisión \\
\hline & & M17 Atribución a falta de esfuerzo \\
\hline
\end{tabular}


Continuación Tabla I.

\begin{tabular}{|l|l|l|}
\hline \multirow{5}{*}{ E3 Optimización } & $\begin{array}{l}\text { D8 Optimización } \\
\text { positivamente }\end{array}$ & M29 Evaluación positiva \\
\cline { 2 - 3 } & $\begin{array}{l}\text { D9 Optimización } \\
\text { preparatoria }\end{array}$ & M30 Transformación rentabilizadora \\
\cline { 2 - 3 } & \multirow{2}{*}{$\begin{array}{l}\text { D10 Optimización } \\
\text { autopotenciadora }\end{array}$} & M27 Control emocional anticipatorio \\
\cline { 2 - 3 } & M23 Atribución internalista del éxito \\
\cline { 2 - 3 } & M26 Autribución a las estrategias \\
\cline { 2 - 3 } & M28 Anticipación constrón proactiva \\
\hline
\end{tabular}

Con atención al modelo, el encuadre de Espontaneidad vital (El) es el que permite que el modo de situarnos ante las situaciones sea implicativo, bien de manera espontánea y natural, o de forma reflexiva y calculadora. El encuadre de Ajuste (E2) faculta a que el enfoque sobre el contexto sea equilibrado cuando lo ostensivo aparece como positivo, mientras que inestable cuando es negativo. El encuadre de Optimización (E3) es responsable de que el enfoque sea constructivo y optimista; o bien, improductivo y pesimista.

Las dimensiones o expresiones de los tres encuadres referidos (Hernández, 2002) a partir de las que una persona se conforma cognitiva y emocionalmente, son las siguientes:

Implicación vital (Dl) es la particularidad cognitiva que permite valorar los aspectos positivos del contexto. Negatividad (D2) es la perspectiva cognitiva que se manifiesta en remarcar los aspectos negativos. Discordancia (D3) es la característica cognitiva a través de la cual aceptamos un hecho como es; o bien, lo distorsionamos a fin de imponer nuestros intereses. Evitación (D4) es la perspectiva cognitiva que nos permite evitar abordar nuestras obligaciones, generando opciones irreales, evitativas.

Defensividad (D5) es la dimensión cognitiva que permite evitar la asunción de responsabilidad al atribuir los éxitos y fracasos a variables externas. Atribución externa (D6) es la perspectiva cognitiva que evita asumir la responsabilidad de los éxitos y fracasos. Operatividad (D7) es la dimensión cognitiva a través de la que se preparan los distintos componentes psicológicos que puedan potenciar buenos resultados. Optimización positivante (D8) es la particularidad cognitiva que faculta la valoración positiva sobre nosotros mismos, y la realidad en general. Optimización preparatoria (D9) es la perspectiva cognitiva a través de la cual se potencia el poder personal, atribuyendo éxitos a la propia responsabilidad. Optimitización autopotenciadora (D10), finalmente, es la dimensión cognitiva por medio de la cual los sujetos tienden a implicarse sin exagerar la función reflexiva. 
En un nivel de análisis y expresión más concretos se encuentran, finalmente, los 30 moldes o factores de primer orden, que constituyen las estrategias cognitivo-emocionales peculiares y específicas con las que los sujetos enfocan, reaccionan o interpretan la realidad (Hernández, 2002). En otras palabras, la competencia que cada uno de nosotros tiene para relacionarse con la realidad en condiciones de autoimplicación, se puede representar en tres grandes encuadres focales (espontaneidad vital, ajuste y optimización). En un nivel de análisis intermedio, los tres encuadres se pueden expresar en diez dimensiones, relacionadas con el grado de adecuación con el que usamos ciertas estrategias cognitivas. Finalmente, estas diez dimensiones se manifiestan en treinta moldes mentales que responden a sendas estrategias cognitivo-emocionales que refieren los formatos específicos a través de los cuales anticipamos, reaccionamos, encauzamos, evaluamos, explicamos o resolvemos situaciones egoimplicativas (Hernández, 2010).

\subsection{Densidad argumentativa}

A través del concepto de densidad argumentativa se ha pretendido referir el producto discursivo al cual es capaz de llegar un determinado hablante, en lo que concierne al nivel macroestructural del mismo.

Asumimos que -en dependencia del tipo de estrategias-cognitivo emocionales que active un determinado hablante- se conformará un tipo de discurso con mayor o menor grado de coherencia global determinada por la estructura textual del mismo y las relaciones de sus partes, potencialmente medible en términos de densidad de la textura argumental del entramado discursivo.

Se han supuesto, en consecuencia, variables normativas para el establecimiento de la densidad argumentativa del producto discursivo. Tal cual se pretende representar en la Figura 1, con un ejemplo de fantasía, se sugieren como marcadores de la densidad argumentativa (Noemi, 2013; Noemi, 2014; Hammer y Noemi, 2015): el número de $\mathrm{P}$ (roposiciones) por secuencia; el número de support por secuencia; el número de explain por secuencia; y el número de justify por secuencia.

(P)roposición, representa el contenido semántico de una oración y, por tanto, constituye la unidad básica del nivel inferior de la macroestructura. Support representa un apoyo argumental de la (P)roposición, por lo que supone por parte del hablante mayor compromiso inferencial/representacional y ocupa un lugar de mayor profundidad macroestructural.

Explain constituye una explicación extraída a partir del estado de mundo discursivo expuesto por la (P)roposición y avalada por support, y supone en consecuencia actividad cognitiva de mayor envergadura. Justify, finalmente, representa una justificación extraída a partir del mundo discursivo generado por 
(P)roposición, support y explain, y supone el mayor esfuerzo inferencial asociado a este tipo de discurso, al igual que la máxima profundidad macroestructural asociada a este tipo de discurso (Noemi, 2013; Noemi, 2014; Hammer y Noemi, 2015).

Los significantes support, explain y justify han sido generados de manera automática por el software Atlas.ti.

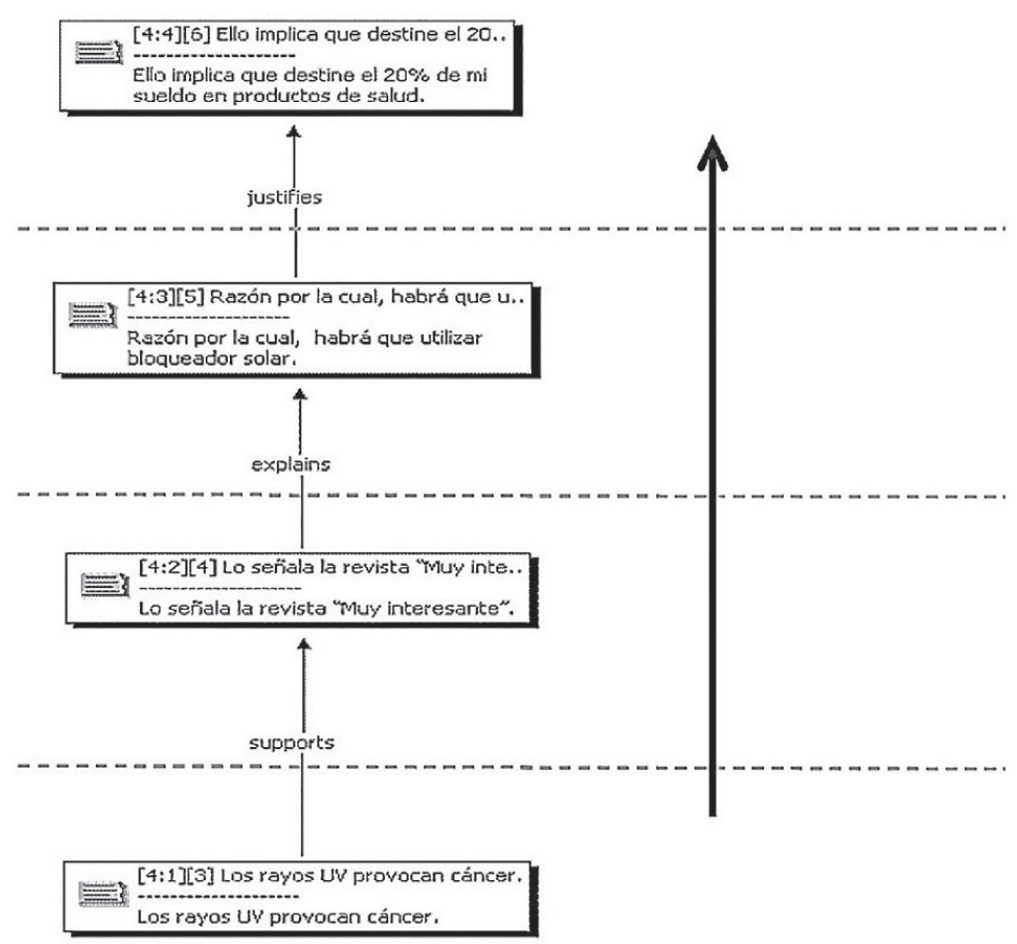

Justify

Explain

Support

Figura1. Representación de niveles de densidad argumentativa.

\section{METODOLOGÍA}

El trabajo siguió una metodología mixta, cuantitativa y cualitativa. Desde la primera perspectiva, se aplicó el test Moldes (Hernández, 2010) para determinar la frecuencia de uso de determinados moldes cognitivo-emocionales. Desde la segunda, se empleó el Dilema III del cuestionario de problemas socio-morales DIT (Rest, 2013), a fin de que los sujetos de la muestra generaran un texto con secuencias argumentativas, a partir del cual se pudiera describir el grado de densidad 
argumentativa alcanzado, por medio del software Atlas.ti.

El test Moldes es un cuestionario que comprende 87 ítems orientados a describir estrategias socio-cognitivas empleadas por los sujetos que se enfrentan a una tarea autoimplicativa. Su principal objetivo es conocer los modos habituales con los que una persona se enfrenta a la realidad y las maneras como la interpreta y valora su relación con ella.

El cuestionario de problemas socio-morales D.I.T. (Rest, 2013), basado en la teoría de Kohlberg (1992), busca establecer el razonamiento moral a través del planteamiento de 6 dilemas sociomorales. Sin embargo, en este trabajo sólo se utilizó el Dilema III, denominado 'El preso evadido', ya que fue empleado como una estrategia que permitiera elicitar discurso argumentativo, en atención de la similar naturaleza egoimplicativa que el test Moldes.

Así, mientras que el test Moldes ayudó a discriminar las estrategias cognitivo-emocionales empleadas por los sujetos, el Dilema III del cuestionario D.I.T. permitió el levantamiento de información necesaria para establecer el grado de densidad argumentativa de las conductas discursivas asociadas.

Ambas pruebas son de carácter colectivo y autoadministrable. En una misma sesión, se aplicó en primer lugar el test Moldes. Inmediatamente, se procedió a enfrentar a los sujetos ante el Dilema III (Rest, 2013), solicitándoles a continuación y por escrito un punto de vista argumentado en relación con el dilema expuesto. La aplicación de ambos instrumentos demandó aproximadamente una hora de atención.

La muestra abarcó a 78 estudiantes de primero, segundo y tercer año de la carrera de Castellano y Filosofía de la Universidad de La Serena, con edades de entre los 18 y 23 años, y una media de 21 años.

A partir de los datos obtenidos se realizó un muestreo intencionado (Miles y Huberman, 1994), seleccionando aquellos protocolos del test Moldes que evidenciaron las mayores diferencias cuantitativas a nivel intraescala. La corrección de los 78 perfiles se ejecutó de manera on-line a través de la plataforma www.teacorrige. com, la que arrojó como resultado una unidad de observación compuesta por 20 sujetos, correspondientes a los puntajes más dispares entre sí.

Enseguida, se procedió a realizar el análisis textual de las respuestas de los 20 sujetos obtenidas a partir del dilema, por medio del programa Atlas.ti., con la finalidad de determinar el grado de densidad argumentativo evidenciado.

La información cuantitativa de los protocolos del test Moldes se cruzó finalmente con los resultados del análisis textual del Dilema III del cuestionario DIT. Esto permitió describir y relacionar la tendencia en el uso de ciertas estrategias cognitivo-emocionales y la densidad argumentativa alcanzada por los 20 sujetos que compusieron la unidad final de observación. 


\section{ANÁLISIS}

Con el propósito de proceder al análisis cuantitativo de los resultados, a partir de los resultados arrojados por el test Moldes se establecieron dos grupos conformados por 10 sujetos cada uno, tipificados como Grupos 1 y 2, respectivamente. El Grupo 1 corresponde a aquellos sujetos que obtuvieron un rendimiento óptimo en el test Moldes; el Grupo 2, en cambio, quedó constituido por quienes obtuvieron un rendimiento deficiente. Las categorías óptimo/deficiente representan los extremos de la diferencial discriminatoria que arroja el test y que recorre la gradiente óptimo, alto, normal, bajo y deficiente, según sea el molde evaluado.

Luego de la conformación de los grupos 1 y 2, se seleccionó a un sujeto prototípico de cada grupo, etiquetados como SF9 (sujeto femenino número 9) y SF7 (sujeto femenino número 7), quienes son representativos de rendimiento óptimo y deficiente, respectivamente.

A fin de facilitar la interpretación, se procedió a continuación a referir el grado de densidad argumentativa alcanzado por cada uno de los sujetos prototípicos, seguido de una descripción del grado de densidad argumentativa logrado por la totalidad de los miembros pertenecientes a cada grupo.

Según es posible observar, el sujeto rotulado como SF9 (ver Figura 2) quien manifiesta un nivel óptimo en el test Moldes genera discurso con considerable densidad argumentativa. A partir de la tarea escritural gatillada por el Cuestionario D.I.T., en efecto, se comprueba la existencia de $10 \mathrm{P}$ (proposiciones), soportadas por 11 inferencias en el nivel discursivo de support.

Algunas de ellas, según se observa, muestran diferentes expresiones relacionales macroestructurales, particularmente la P(roposición) 2:1 que se vincula con dos proposiciones en el nivel de support (2:3 y 2:2), respectivamente; y la P(roposición) 2:9 que se relaciona en el nivel de support con 2:10 y 2:11.

El sujeto SF9 alcanza el tercer nivel de densidad argumentativa en cuatro ocasiones; en primer lugar, a través de la $\mathrm{P}$ (roposición) 2:1 que se prolonga macroestructuralmente hasta el nivel de explain en la proposición 2:4; por medio de la $\mathrm{P}$ (roposición) 2:12 que se extiende hasta 2:14; mediante la $\mathrm{P}$ (roposición) 2:15 que proyecta hasta 2:18; y finalmente, a través de la $\mathrm{P}$ (roposición) 2:24 que se prolonga semánticamente hacia 2:26. El sujeto SF9 muestra densidad igualmente mediante el vínculo de cohesión que establecen las proposiciones 2:3 y 2:2 del nivel de support con la proposición 2:4 del nivel de explain. 


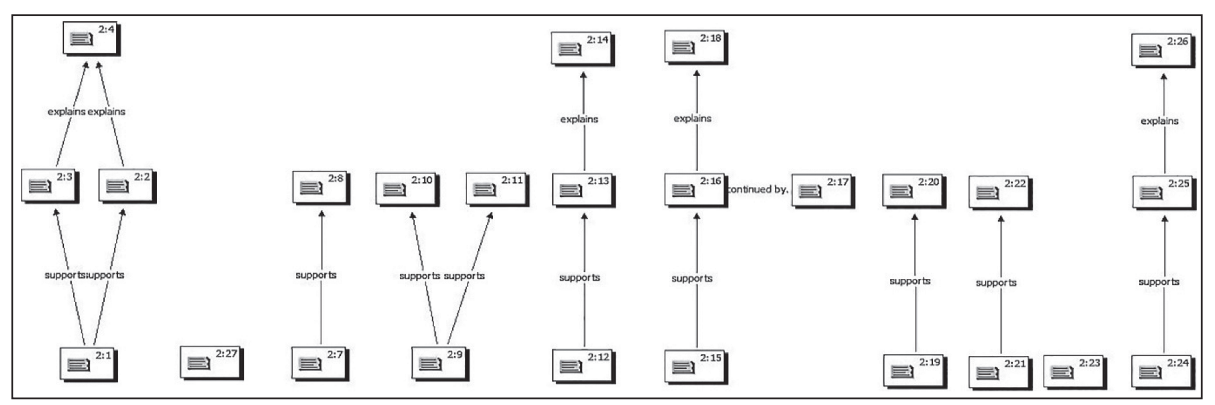

Figura 2. Densidad argumentativa sujeto SF9 del Grupo 1

(Rendimiento adecuado).

Como es posible observar, el sujeto SF9 es representativo del Grupo 1 de acuerdo con los datos que se ilustran en la Tabla II, la que provee información sobre la densidad argumentativa que alcanzan los sujetos de buen rendimiento en el test Moldes.

Según se comprueba, la muestra ofrece resultados uniformes para el grupo en su conjunto. Efectivamente, una lectura vertical de la Tabla II permite observar que la totalidad de los sujetos pertenecientes al Grupo 1 realizan las inferencias necesarias que les permiten generar discurso y alcanzar completamente los niveles de densidad argumentativa representados por support y explain.

Por su parte, siete de ellos (SF16, SF44, SM53, SM21, SM22, SM28 y SM55) efectúan las inferencias que les permiten lograr el nivel máximo de densidad representado por justify. Como se observa, a su vez, cuatro sujetos generan 2 proposiciones en este nivel superior (SM53, SM22, SM28 y SM55), lo que igualmente puede ser interpretado como un rasgo de mayor densidad.

Una lectura horizontal de la Tabla II nos permite observar que el promedio de proposiciones del nivel basal de generación discursiva $\mathrm{P}$ (roposición) alcanza la cifra de 5.8 unidades; el promedio de proposiciones del nivel de support llega a 5 unidades; el promedio de proposiciones en explain es de 2.6; en tanto que el de justify alcanza las 1.1 proposiciones.

Se delinea de este modo una pirámide discursiva con abundante generación de proposiciones en la base, las que van progresivamente disminuyendo a medida que aumenta el compromiso inferencial representado en la densidad macroestructural. 
Tabla II. Densidad Argumentativa Grupo 1.

\begin{tabular}{|l|c|c|c|c|c|c|c|c|c|c|}
\hline \multicolumn{10}{|c|}{ Sujetos Grupo 1 } \\
\hline & SF9 & SF16 & SF44 & SM53 & SM21 & SM22 & SM28 & SM55 & SF30 & SF38 \\
\hline \multicolumn{10}{|c|}{} & Justify \\
\hline Explain & 5 & 3 & 5 & 2 & 1 & 3 & 3 & 2 & 2 & 1 \\
\hline Support & 10 & 4 & 6 & 6 & 3 & 4 & 7 & 6 & 3 & 1 \\
\hline (P)roposición & 10 & 5 & 7 & 8 & 4 & 3 & 6 & 8 & 3 & 4 \\
\hline
\end{tabular}

Por otra parte, es posible observar que el sujeto SF7 (sujeto femenino número 7), quien manifiesta un nivel deficiente en el test Moldes y constituye consecuentemente un representante prototípico del Grupo 2, alcanza un nivel de densidad argumentativa bajo (ver Figura 3). En efecto, a partir de la exigencia escritural representada en el Cuestionario D.I.T. SF7 sólo genera 3 P(roposiciones), soportando discursivamente sólo una de ellas en el entramado argumental en el nivel de support (1:1 hasta 1:2).

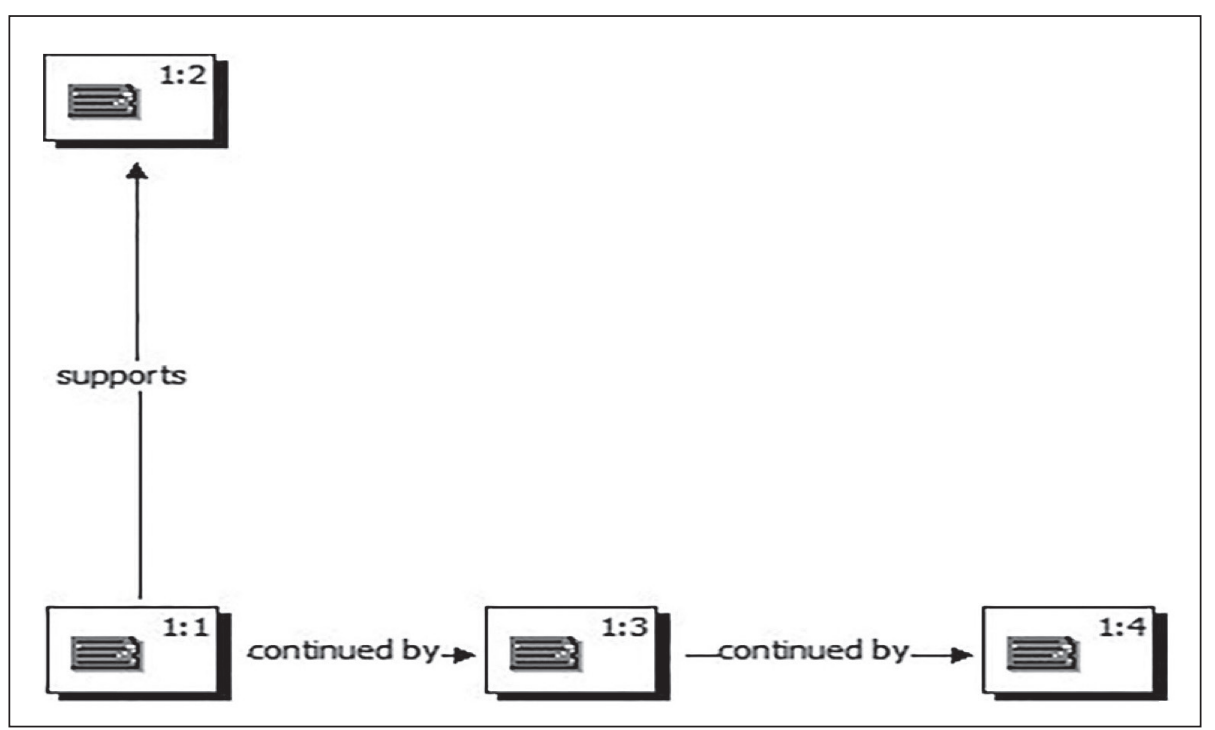

Figura 3. Densidad argumentativa sujeto SF7 del Grupo 2

(Rendimiento inadecuado). 
El sujeto SF7 representa la conducta discursiva generalizada del Grupo 2 (ver Tabla III); es decir, la de aquellos individuos de bajo rendimiento en el test Moldes. La lectura vertical de la Tabla III permite observar que la totalidad de los sujetos pertenecientes a este grupo sólo generan discurso en el nivel de $\mathrm{P}$ (proposición) y efectúan las inferencias mínimas para acceder al nivel Support, a excepción de un individuo, (SF70) quien -en efecto- genera una proposición en el nivel de explain y sobrepasa significativamente la media, al generar cinco proposiciones en el nivel de support.

Una lectura horizontal de la Tabla III nos permite advertir que, al contrario de lo observado en el Grupo 1, en este caso el promedio de proposiciones del nivel $\mathrm{P}$ (roposición) alcanza la cifra de 2.8 unidades; el promedio de proposiciones del nivel de support es de 1.9 unidades; en tanto que el promedio de proposiciones en explain es de 0.1. Como se ha esbozado, el Grupo 2 se caracteriza por la ausencia de proposiciones en los niveles superiores de densidad argumentativa (explain y justify).

Se delinea en este caso un rectángulo discursivo con mediana generación de proposiciones en la base, las que desaparecen abruptamente a medida que aumenta el compromiso inferencial representado en los niveles superiores de densidad macroestructural.

Tabla III. Densidad Argumentativa del Grupo 2.

\begin{tabular}{|l|c|c|c|c|c|c|c|c|c|c|}
\hline \multicolumn{10}{|c|}{ Sujetos Grupo 2 } \\
\hline & SF7 & SF33 & SF41 & SF42 & SF54 & SF58 & SF72 & SF67 & SF61 & SF70 \\
\hline Justify & & & & & & & & & & \\
\hline Explain & & & & & & & & & & 1 \\
\hline Support & 1 & 1 & 2 & 2 & 2 & 2 & 1 & 2 & 1 & 5 \\
\hline (P)roposición & 1 & 4 & 3 & 3 & 3 & 2 & 3 & 3 & 2 & 4 \\
\hline
\end{tabular}

\section{HALLAZGOS}

Los resultados obtenidos permitieron determinar que los sujetos pertenecientes al Grupo 1, es decir aquellos que alcanzan un nivel considerado como óptimo en el test Moldes y consecuentemente detentan adecuadas estrategias psico-sociales a la hora de afrontar tareas de autoimplicación, son capaces de generar discurso con mayor grado de densidad argumentativa, expresado tanto en el promedio del 
número de proposiciones por nivel de inferencias, como por la naturaleza de las mismas (ver Tabla II).

En igual sentido, ha sido posible observar que los sujetos pertenecientes al Grupo 2, a saber aquellos que sólo lograron un nivel considerado como deficiente en el test Moldes y por tanto quienes manifiestan inadecuadas estrategias psicosociales al abordar trabajos autoimplicativos, sólo son capaces de generar discurso con un nivel bajo de densidad argumentativa en términos del número de proposiciones, permaneciendo además preferentemente en el nivel de $\mathrm{P}$ (roposición) y alcanzando exclusivamente inferencias a nivel de support (ver Tabla III).

En conformidad con los datos, resulta posible señalar que las estrategias cognitivo-emocionales específicas que favorecen conductas verbales con alta densidad argumentativa (Hernández, 2002) son: implicancia directa (M1), es decir, la capacidad de aproximarse a un problema de forma implicativa; anticipación constructiva previsora (M28), esto es, la facultad para anticipar los pros y contras del enfrentamiento a una situación determinada; precisión-supervisión (M16), a saber, la disposición mental para supervisar y consolidar una tarea; evaluación positiva (M29), vale decir, la estrategia que permite focalizar aspectos positivos y obviar los negativos de un hecho; transformación rentabilizadora (M30), es decir, la capacidad que permite transformar los problemas en posibles ventajas; autoconvicción volitiva (M25), esto es, la capacidad para implicarse activamente en una determinada situación; control emocional anticipatorio (M27), a saber, la facultad para prever situaciones adversas; atribución internalista del éxito (M23), vale decir, la capacidad derivada de la autoconfianza que permite remarcar logros frente a una situación dada; atribución a las estrategias (M24), esto es, la disposición que faculta atribuir los éxitos y fracasos a las estrategias empleadas; automotivación proactiva (M26), es decir, la estrategia que predispone a pensar en los logros y las ventajas para procurar disminuir las dificultades; y finalmente, anticipación constructiva previsora (M28), a saber, la competencia para anticipar los pros y contras del enfrentamiento a una situación determinada (ver Tabla IV y Anexo 1).

Por el contrario, de acuerdo con lo observado, las características cognitivoemocionales que atentan en contra de o inhiben las conductas verbales con alta densidad argumentativa (Hernández, 2002) son: hipercontrol anticipatorio (M3), es decir, la disposición exagerada de pretender controlar con el pensamiento todo el entorno; anticipación aversiva (M5), vale decir, la condición mental que aumenta las consecuencias negativas de algún problema; anticipación devaluativa (M11), esto es, la disposición para devaluar los hechos; hostiligencia (M7), a saber, la disposición mental que presume daños infringidos por los demás; afrontamiento borroso (M4), vale decir, la condición que conduce a suscitar incertidumbre, confusión e indecisión frente a un problema; inflación-decepción (M8), esto es, la capacidad para imaginar de forma exagerada y fantasiosa el contexto; imantación por lo imposible (M10), a saber, la disposición que impide la consideración y con- 
secución de metas realistas; disociación emocional (M12), esto es, la facultad para evitar autoimplicarse; atribución social del éxito (M21), es decir, la incapacidad para considerar los méritos propios; y, finalmente, atribución mágica (M22), esto es, la disposición para atribuir los éxitos o fracasos a variables tales como el destino, o la suerte (ver Tabla IV y Anexo 1).

Tabla IV. Estrategias cognitivo-emocionales que facultan o limitan la densidad argumentativa.

\begin{tabular}{|l|l|}
\hline \multicolumn{2}{|c|}{ Estrategias cognitivo-emocionales } \\
\hline A favor de densidad argumentativa & En contra de densidad argumentativa \\
\hline - Implicancia directa (M1) & - Hipercontrol anticipatorio (M3) \\
- Anticipación constructiva previsora (M28) & - Anticipación aversiva (M5) \\
- Precisión-supervisión (M16) & - Anticipación devaluativa (M11) \\
- Evaluación positiva (M29) & - Hostiligencia (M7) \\
- Transformación rentabilizadora (M30) & - Afrontamiento borroso (M4) \\
- Autoconvicción volitiva (M25) & - Inflación-decepción (M8) \\
- Control emocional anticipatorio (M27) & - Imantación por lo imposible (M10) \\
- Atribución internalista del éxito (M23) & - Disociación emocional (M12) \\
- Atribución a las estrategias (M24) & - Justificación de los fallos (M18) \\
- Automotivación proactiva (M26) & - Atribución social del éxito (M21) \\
- Anticipación constructiva previsora (M28) & - Atribución mágica (M22) \\
\hline
\end{tabular}

En términos generales, se observa que los sujetos que manifiestan alta densidad argumentativa, expresada en un entramado discursivo de fuerte cohesión y coherencia macroestructural, tienen una valoración positiva de sí mismos y capacidad para anticipar éxitos y atribuirlos a su propia responsabilidad, condición que les favorece a la hora de aventurar buenos resultados frente a una tarea o problema.

Por otra parte, los individuos que evidencian baja densidad argumentativa, observada en una urdimbre discursiva de poca coherencia, procuran evitar el afrontamiento de problemas; o bien, en caso de abordarlos, lo hacen de manera negativa, evitando atribuir los éxitos o fracasos a su propia responsabilidad.

\section{CONCLUSIÓN}

El trabajo ha permitido evidenciar el vínculo entre estrategias cognitivo-emocionales de estudiantes universitarios, y el grado de densidad textual que alcanzan 
en la producción de discurso argumentativo. Particularmente, se pudo esbozar el dominio (Fodor, 1986) de las estrategias cognitivo-emocionales en relación con el grado de densidad textual argumentativa alcanzada en sus productos discursivos.

Los resultados indicaron que los sujetos que tienden a la utilización de moldes más operativos, realistas, positivos, de afrontamiento de la realidad problemática, de encaje emocional y de capacidad optimizadora de las posibilidades tienden a presentar un mayor grado de densidad argumentativa.

Por otra parte, los datos han permitido establecer que aquellos sujetos que funcionan con moldes más inoperantes, no realistas, negativistas, de desconexión con la realidad y de ausencia de encaje emocional, tienden a obtener un menor rendimiento en términos de un discurso argumentativamente denso.

Si consideramos que los moldes mentales pueden concebirse como modos habituales con los que una persona se enfrenta cognoscitiva y afectivamente a la realidad y con los que interpreta y valora su relación con ella, su valor funcional devela la importancia tanto de las competencias intelectivas como emocionales a la hora de trabajar sobre el potencial de aprendizaje asociado (Capote et al., 2007).

En este sentido, el trabajo ha arrojado evidencia empírica respecto de aquellas estrategias cognitivo-emocionales que se vinculan con tipos de productos discursivos esperables, aspecto que contribuye igualmente a considerar las emociones como una variable a la hora de describir la capacidad y el producto del acto de habla de argumentar.

Si tenemos en consideración, por otra parte, que ya se han identificado empíricamente aquellos moldes mentales que guardan relación con un mejor rendimiento académico entre universitarios (Hernández, 2005), los resultados obtenidos por este trabajo abren la posibilidad de elaborar planes remediales de intervención para tratar con sujetos de baja competencia discursiva argumentativa. 


\section{ANEXOS}

Anexo 1. Especificación moldes.

\begin{tabular}{|c|c|c|}
\hline M1 & Implicancia Directa & Molde consistente en activar la fuerza de voluntad para implicarse en las situaciones \\
\hline M2 & Hiperanálisis & Molde consistente en exagerar el distanciamiento con la realidad, autoinhibiéndose \\
\hline M3 & Hipercontrol anticipatorio & Molde consistente en administrarse ánimo, anticipándose autorrecompensas \\
\hline M4 & Afrontamiento borroso & Molde consistente en plantearse metas difusas \\
\hline M5 & Anticipación aversiva & Molde consistente en anticipar problemas y fracasos \\
\hline M6 & Evaluación selectiva negativa & Molde consistente en relevar lo negativo \\
\hline M7 & Hostiligencia & Molde consistente en atribuir mala intención a los congéneres \\
\hline M8 & Inflación-decepción & Molde consistente en plantearse metas desmedidas que conducen a decepción \\
\hline M9 & Focalización en las carencias & Molde consistente en relevar lo carente \\
\hline M10 & Imantación por lo imposible & Molde consistente en apegarse a conductas inviables \\
\hline M11 & Anticipación devaluativa & Molde consistente en desvalorizar resultados para evitar desencanto \\
\hline M12 & Disociación emocional & Molde consistente en distanciarse, autoadministrando desinterés \\
\hline M13 & Reclusión & Molde consistente en distanciarse de la realidad para evitar frustraciones \\
\hline M14 & Oblicuidad cognitiva & Molde consistente en desviar la relevancia cognitiva \\
\hline M15 & Anticipación esfuerzo-costo & Molde consistente en reflexionar de manera confiada y operativa \\
\hline M16 & Precisión y supervisión & Molde consistente en definir y supervisar las conductas \\
\hline M17 & Atribución a la falta de esfuerzo & Molde consistente en autoatribuir los fracasos a la falta de esfuerzo \\
\hline M18 & Justificación de los fallos & Molde consistente en autojustificar los fracasos \\
\hline M19 & Desplazamiento emocional & Molde consistente en desplazar la responsabilidad hacia otros \\
\hline M20 & Atribución al temperamento & Molde consistente en atribuir el éxito al temperamento propio \\
\hline M21 & Atribución social del éxito & Molde consistente en atribuir los éxitos a los demás \\
\hline M22 & Atribución mágica & Molde consistente en atribuir los éxitos o fracasos a fuerzas desconocidas \\
\hline M23 & Atribución internalista del éxito & Molde consistente en autoatribuirse los éxitos \\
\hline M24 & Atribución a las estrategias & Molde consistente en atribuir el éxito a sus estrategias \\
\hline M25 & Autoconvicción volitiva & Molde consistente en activar la fuerza de voluntad para implicarse en las situaciones \\
\hline M26 & Automotivación proactiva & Molde consistente en administrarse ánimo, anticipándose autorrecompensas \\
\hline M27 & Control emocional anticipatorio & Molde consistente en prever posibles reacciones emocionales \\
\hline M28 & Anticipación constructiva previsora & Molde consistente en anticipar problemas y fracasos \\
\hline M29 & Evaluación positiva & Molde consistente en administrarse autoestima \\
\hline M30 & Transformación rentabilizadora & Molde consistente en rentabilizar positivamente lo negativo \\
\hline
\end{tabular}

\section{REFERENCIAS}

Beck, Aaron. (1974). The development of depression: Idiosyncratic content and cognitive distortions. Archives of General Psychiatric 8, 324-333.

Capote, María; García, Victorio y Hernández, Pedro. (2002). Matemáticas y moldes cognitivos. Evaluación e intervención psicoeducativa 1(8-9), 175-204.

Capote, María; Fernández, Andrés y Hernández, Pedro. (2007). Moldes mentales en el proceso de aprendizaje. Revista de orientación educativa, 39, 31-46.

Fodor, Jerry. (1986). La modularidad de la mente. Madrid: Ediciones Morata. Graesser, Arthur; Singer, Murray y Trabasso, Tom. (1994). Constructing inferences during narrative text Comprehension. Psychological Review, 101, 371-395. Hastings, Arthur. (1963). A Reformulation of the Modes of Reasoning in Argumentation. Ph.D. dissertation. Northwestern University, Illinois. 
Hammer, Liam y Noemi, Cristián. (2015). Relación entre pensamiento crítico y complejidad discursiva en estudiantes universitarios. Onomazein, 32, DOI 10.7764/onomazein.32.1

Hample, Dale. (2005). Arguing: Exchanging Reasons Face to Face. Mahwah, NJ: Erlbaum.

Hample, Dale; Warner, Ben y Young, Dorian. (2009). Framing and Editing Interpersonal Arguments. Argumentation, 23, 21-37, DOI 10.1007/s 10503-0089107-x

Hernández, Pedro. (1991). Psicología y educación: corrientes actuales y teorías aplicadas. México: Trillas.

Hernández, Pedro. (2002). Los moldes de la mente: más de la inteligencia emocional. La Laguna, Tenerife: Tafor publicaciones.

Hernández, Pedro. (2005). ¿Puede la inteligencia emocional predecir el rendimiento? Potencial predictor de los moldes mentales. Revista interuniversitaria de formación del profesorado, 19(3), 45-62.

Hernández, Pedro. (2006). Inteligencia emocional y moldes mentales en la explicación del bienestar subjetivo. Ansiedad y estrés, 12(2-3), 151-166.

Hernández, Pedro. (2007). Moldes mentales y emociones en el rendimiento de la memoria. Aula, 166, 21-26.

Hernández, Pedro. (2010). Test de estrategias cognitivo-emocionales. Madrid: Tea ediciones.

Hernández, Pedro y Rodríguez, Heriberto. (2006). Success in chess mediated by mental molds. Psicothema 18(4), 704-710.

Jélvez, Lorena. (2008). Esquemas argumentativos en textos escritos: un estudio descriptivo en alumnos de tercero medio de dos establecimientos de Valparaíso. Cyberhumanitatis, 45.

Kienpointner, Manfred. (1987). Towards a typology of argumentative schemes. In F. Van Eemeren, R. Grootnedorst, J. A. Blair, \& C. A. Willard (Eds.), Argumentation: Across the lines of discipline. Proceedings of the conference on argumentation (pp. 275-287). Providence: Foris Publications.

Kohlberg, L. (1992). Psicología del desarrollo moral. Bilbao: Desclée de Brouwer.

Miles, Matthew y Huberman, Alan. (1994). Qualitative data analysis: A sourcebook of new methods. London: Sage.

Marinkovich, Juana. (2007). La interacción argumentativa en el aula: fases de la argumentación y estrategias de cortesía verbal. En C. Santibánez, y B. Riffo (Eds.), Estudios en Argumentación y Retórica. Teorias contemporáneas y aplicaciones (pp. 227-252). Concepción: Editorial Universidad de Concepción.

Noemi, Cristián. (2013). Aproximación teórica a la noción de complejidad argumentativa. Logos: revista de lingüistica, filosofía y literatura, 22, 2, 256-271.

Noemi, Cristián. (2014). Un modelo de representación de complejidad argumentativa: el discurso de Friedman. Linguagem em (Dis)curso, 15, 2, 305-320. 
Padilla, Constanza; Douglas, Silvina y López, Esther. (2010). Competencias argumentativas en la alfabetización académica. Revista @tic, revista d'Innovació educativa 4, 2-12.

Perelman, Chaim y Olbrechts-Tyteca, Lucie. (1989). Tratado de la argumentación: la nueva retórica. Madrid: Gredos.

Rest, James. (2013). Cuestionario de Problemas Socio-morales D.I.T. Madrid: Darwf.

Santibáñez, Cristián (2016). Mindreading, representación, inferencia y argumentación. En Santibánez, C. (Compilador) Ecología argumentative universitaria: desde la realidad a los conceptos (pp. 19-52). Concepción, Cosmigonon Ediciones.

Tomasello, Michael. (1999). Los origenes culturales de la cognición humana. Buenos Aires: Amorrortu Editores.

Van Dijk, Teun. (1992). La ciencia del texto. Barcelona: Ediciones Paidós.

Van Dijk, Teun y Kintsch, Walter. (1983) Strategies of comprehension. New York: Academic Press.

Van Eemeren, Frans. (2012). The Role of Logic in Analyzing and Evaluating Argumentation. In J. Ribeiro (Ed.), Inside arguments: logic and the study of argumentation (pp. 139-155). Cambridge: Cambridge Scholars Publishing.

Van Eemeren, Frans; Grootendorst, Rob y Snoeck, Francisca. (2006). Argumentación. Análisis, evaluación, presentación, Buenos Aires: Biblios.

Véliz, Mónica. (1999). Complejidad sintáctica y modo del discurso. Estudios Filológicos, 34, 181-192.

Walton, Douglas. (1996). Argumentation schemes for presumtive reasoning. Mahwah NJ: Lawrence Erlbaum Associated, Inc.

Walton, Douglas. (1997). Appeal to Expert Opinion. University Park: The Pennsylvania State University Press.

Walton, Douglas. (2005). Justification of argument schemes. Australasian Journal Logic, 3, 1-13.

Walton, Douglas; Reed, Chris y Macagno, Fabrizio. (2008). Argumentation schemes. Nueva York: Cambridge University Press. 